ISBN: 978-1-948012-12-6

\title{
AN IMPROVED COGNITIVE RADIO RESOURCE ALLOCATION ALGORITHM BASH ON RELAY COOPERATION
}

\author{
Qi Feng, Lingling Chen* \\ College of Information and Control Engineering, Jilin Institute of Chemical Technology No.45 chengde street, longtan district, jilin city, jilin \\ province, China. \\ *Corresponding Author Email: cll11807900@163.com
}

This is an open access article distributed under the Creative Commons Attribution License, which permits unrestricted use, distribution, and reproduction in any medium, provided the original work is properly cited.

\section{ARTICLE DETAILS}

\section{Article History:}

Received 26 June 2018 Accepted 2 July 2018 Available online 1 August 2018

\section{ABSTRACT}

In this paper, a resource allocation algorithm is designed in relay cooperative cognitive radio networks. In amplify and forward (AF) cooperative communication mode, the goal of the proposed algorithm is to maximize the system capacity. Under the same conditions, the results of simulation show that the signal to interference ratio (SINR) of relay cooperative is better than non-relay cooperative's SINR. The transmission power of relay cooperative is less than non-relay cooperation. The relay cooperation is superior to traditional algorithms in convergence rate, the user's QoS also can be better guaranteed.

\section{KEYWORDS}

Relay Coordination, Power Distribution, Signal to Noise Ratio, Cognitive Radio.

\section{INTRODUCTION}

Nowadays, with the rapid development of wireless communication networks and mobile devices, the contradiction between limitation of spectrum resources and the growing demand of spectrum is becoming significantly conflicting. In order to alleviate the above contradiction. Joseph Mitola was first proposed the concept of cognitive radio (CR) [1,2]. Cognitive radio can grasp the information of the surrounding spectrum by using the spectrum sensing and realize the communication by finding the idle spectrum, which can make use of the idle spectrum to improve the efficiency of the spectrum and simultaneously guarantee the QoS of the authorized user and cognitive user as well as significantly reduce the network energy consumption [3,4]. However, the available spectrum may be different since the different receiving location of users in cognitive radio network. When the transmit power of cognitive user is restricted with the interference temperature, it leads to a reduction in cognitive system capacity [5]. In order to solve this problem, the relay collaboration technology is proposed.

Recently, the relay cooperation technology has attracted much attention. In 2000, assuming that cognitive users are not considered to have interference with authorized users, Jovicic.A.etc. have studied the capacity of cognitive systems in [6]. In, Gastper M. studied the system capacity of cognitive system with limited transmission power, which laid a solid foundation for the research of power allocation in relay cooperation [7]. Hanan Al-Tous studied that it established the power allocation and spectrum allocation into the Stackelberg model at the amplification and forwarding mode and proposed a distributed power acquisition algorithm in presented a new spectrum sensing scheme to achieve almost the best perception performance, which compared with the traditional scheme [8, 9]. The relay node greatly reduced the performance of the system during the mobile process. Reference proposed a relay dynamic selection strategy based on the channel state, which can effectively reduce the interruption probability and increase the diversity gain as well as expand the spectrum utilization ratio [10]. Zhou Mingyue proposed a relay allocation strategy which only considered the interference threshold in [11]. Although the strategy can guarantee the authorized user's QoS, but it cannot guarantee the QoS of the cognitive user.

Through the understanding of the above literature, we can draw the fact that it can effectively overcome the large scale fading through the cooperative communication between the relay points, improve the spectrum utilization, expand the system capacity and reduce the energy consumption of the system. In order to guarantee the QoS of the cognitive users, a relay allocation strategy considering interference temperature limitation and signal to interference ratio is proposed in this paper, which can guarantee the signal-to-noise ratio of both authorized users and cognitive users.

\section{SYSTEM MODEL}

In the cognitive radio system, spectrum sharing, and opportunistic spectrum access are two types of spectrum access [12]. The spectrum sharing means that cognitive users and authorized user can share the same spectrum, and the interference from cognitive users cannot exceed the maximum interference threshold permitted by authorized user.

The opportunity spectrum access method is to make full use of the spectrum sensing technology to detect the transmission state of the authorized user. When the authorized user does not occupy the idle spectrum, the cognitive users can use the idle spectrum. In this chapter, we study the cognitive radio network on the platform of spectrum sharing. As shown Figure 1.

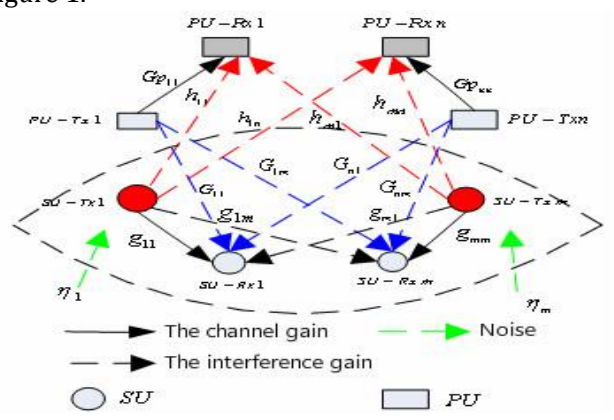

Figure 1: Cognitive network 
In cognitive network with centrol nodes, there are $\mathrm{X}$ cognitive users and $\mathrm{M}$ authorized users, which each relay can only be occupied by a cognitive radio. In the transmission process, there are many kinds of interference. when considering the background noise, the authorized users' interference to the cognitive users and the interference between users. The problem can be expressed as follows

$$
z_{x}=\sum_{x=1}^{X} d_{x y} p_{y}+\sigma_{x}+I_{x}
$$

where $\sigma_{x}$ is background noise. $p_{y}$ denotes the transmission power of cognitive users transmitter. $d_{x y}$ is the interference gain from link y to link x. $I_{x}$ denotes the interference of authorized users to cognitive users. The signal noise ratio in the link $\mathrm{x}$, the problem can be depiced as follows

$$
\gamma_{x}=p_{x}\left|h_{x x}\right|^{2} / z_{x}^{2}
$$

where $p_{x}$ denotes the transmit power of cognitive user. $h_{x x}$ is the average channel gain from transmitter i to receiver on link $\mathrm{x} . Z_{x}$ denotes the total interference in the link $\mathrm{x}$. In practical application, the signal-tonoise ratio (SINR) of cognitive users is greater than the minimum quality of service (QoS) of users, to ensure the QoS of the cognitive user as follows

$$
\gamma_{x} \geq \gamma_{x}^{\min }
$$

where $\gamma_{x}$ denotes the signal-to -noise ratio (SINR) of the cognitive user x. $\gamma_{x}^{\min }$ denotes the minimum signal-to-noise ratio (SINR) to meet the communication requirements. In practical application, the transmission power of the cognitive user is less than the maximum of the transmission power,as follows

$$
0 \leq p_{x} \leq p_{x}^{\max }, x=1,2, \cdots, X
$$

where $p_{x}$ denotes the transmission power of cognitive user, $p_{x}^{\max }$ is the maximum transmit power for cognitive user. In order to ensure QoS of cognitive user, the interference power from each cognitive user should not exceed the authorized users' permissible threshold when communication between users.

$$
p_{x}\left|v_{x m}\right|^{2} \leq A_{x m}
$$

where $v_{x m}$ is the channel gain from the transmitter $\mathrm{x}$ of cognitive user to the receiver m of authorized user. $A_{x m}$ denotes the maximum permissible threshold from transmitter $\mathrm{x}$ of cognitive user to the receiver $\mathrm{m}$ of authorized user. For achieving maximizing the capacity of each cognitive user, and satisfying the constraints of formula 3 , formula 4 , and formula 5 , the mathematical model can be expressed as follows

$$
\begin{aligned}
& \max \sum_{x=1}^{X} \log \left(1+p_{x}\left|h_{x x}\right|^{2} / z_{x}^{2}\right) \\
& \text { s.t. }\left\{\begin{array}{c}
0 \leq p_{x} \leq p_{x}^{\max } \\
p_{x}\left|v_{x m}\right|^{2} \leq A_{x m} \\
\gamma_{x} \geq \gamma_{x}^{\min }
\end{array}\right.
\end{aligned}
$$

\section{Relay cooperation system model}

In order to better satisfy the users' QoS and improve the system capacity in the mode of the relay cooperation is choose in cognitive radio network. In the cognitive network (CR) of the relay cooperation, the relay users $r$ must satisfy the constrain of the non-relay cognitive user $x$, that is, the transmission power cannot exceed the relay user's permissible transmit maximum threshold and the interference power from relay cognitive users cannot exceed the permissible threshold of the authorized user , such as formula (7), formula(8).

$$
0 \leq p_{r} \leq p_{r}^{\max }
$$

where $p_{r}$ denotes the transmit power of relay cooperation. $p_{r}^{\max }$ denotes the maximum transmission power. The problem can be depicted as follows

$$
p_{r}\left|v_{r m}\right|^{2} \leq B_{r m}
$$

where $v_{r m}$ is the channel gain between the relay $r$ and the authorized user $m . B_{r m}$ is the maximum interference threshold of the authorized user $\mathrm{m}$. In half-duplex mode, each transmission cycle is divided into two time slots. The source node broadcasts its information in first time slot, and the relay node and destination node receive the broadcast information. The relay node sends information to the destination node in second time slot. Therefore, when each cognitive user $\mathrm{x}$ is communicate with other by assisting relay $r$, the system capacity is depicted as follows

$$
C_{x r}=\frac{1}{2} \log \left(1+\gamma_{x d}+\gamma_{x r} \gamma_{r d} /\left(1+\gamma_{x r}+\gamma_{r d}\right)\right)
$$

Let $\gamma_{x d}=p_{x}\left|h_{x x}\right|^{2} / z_{x}^{2}, \gamma_{r d}=p_{r}\left|G_{r x}\right|^{2} / z_{d}^{2}$, and $\gamma_{x r}=p_{x}\left|g_{x r}\right|^{2} / z_{x}^{2}$, where $G_{r x}$ is channel gain between the transmitter of relay and the receivers of cognitive user and $g_{x r}$ is channel gain between the transmitter of relay and the transmitters of cognitive users. In order to maximize system capacity, the model can be depiced as follows

$$
\begin{gathered}
\max \sum_{x=1}^{X} \sum_{r=} \frac{1}{2} \log \left(C_{x r}\right) \\
\text { s.t }\left\{\begin{array}{l}
0 \leq p_{x} \leq p_{x}^{\max } \\
p_{x}\left|v_{x m}\right|^{2} \leq A_{x m} \\
0 \leq p_{r} \leq p_{r}^{\max } \\
p_{r}\left|v_{r m}\right|^{2} \leq B_{r m} \\
\gamma_{x} \geq \gamma^{\min }
\end{array}\right.
\end{gathered}
$$

\section{OPTIMIZED MODEL}

\subsection{Optimization Model of non-relay Cooperative system}

According to the convex optimization theory, we can find that the optimization problem (6) is a non-convex optimization problem, which is transformed it into a convex optimization problem, the problem can be depicted as follows

$$
\begin{gathered}
\min -\sum_{x=1}^{X} \log \left(1+p_{x}\left|h_{x x}\right|^{2} / z_{x}^{2}\right) \\
\text { s.t }\left\{\begin{array}{c}
0 \leq p \leq p_{x}^{\max } \\
p_{x}\left|v_{x m}\right|^{2} \leq A_{x m} \\
\log (1+\gamma) \geq \log \left(1+\gamma_{x}^{\min }\right)
\end{array}\right.
\end{gathered}
$$

\subsection{Relay cooperative optimization model}

The objective function has two variables $p_{x}$ and $p_{r}$ in (10)., the range of $p_{r}$ can be achieved by the constraints (7) and (8).

$$
p_{r}^{*}=\min \left(p_{r}^{\min }, B_{r m} /\left|v_{r m}\right|^{2}\right)
$$


Thus, the above model (10) can be represented as

$$
\begin{aligned}
& \min -\sum_{x=1}^{X} \sum_{r}^{R} \frac{1}{2} \log \left(1+r_{x d}+r_{x r} r /\left(1+r^{*}+r_{x r}\right)\right) \\
& \text { s.t }\left\{\begin{array}{c}
0 \leq p \quad \max _{x} \\
p_{x}\left|v_{x m}\right|^{2} \leq A_{x m} \\
\log \left(1+{ }_{x}\right) \leq \log \left(1+\gamma_{x}^{\min }\right)
\end{array}\right.
\end{aligned}
$$

where $r^{*}=p_{r}^{*}\left|G_{r x}\right|^{2} / z_{r}^{2}$.

\section{POWER ALLOCATION ALGORITHM}

\subsection{Non-Relay Cooperative Power Allocation Algorithm}

It is not difficult to find that Lagrange function of the problem (11) as

$$
\begin{aligned}
L\left\{p_{x},\right. & \left.\lambda_{m}, \mu_{x}, \varphi_{x}\right\}=-\sum_{x=1}^{X} \log \left(1+p_{x}\left|h_{x x}\right|^{2} / z_{x}^{2}\right)+\sum_{x} \lambda_{x}\left(p_{x}-p_{x}^{\max }\right) \\
& +\sum_{x} \varphi_{x}\left(\log \left(1+\gamma_{x}\right)-\log \left(1+\gamma_{x}^{\min }\right)\right) \\
& +\sum_{m} \mu_{m}\left(p_{x}\left|v_{x m}\right|^{2}-A_{x m}\right)
\end{aligned}
$$

where the function $L$ is a convex function of $p_{x}$, let $\partial L / \partial_{x}=0$. The optimal solution can be depicted as

$$
p_{x}^{*}=\left[\left(1+\varphi_{x}\right) /\left(\lambda_{x}+\mu_{m}\left|v_{x m}\right|^{2}\right)-z_{x}^{2} /\left|h_{x x}\right|^{2}\right]^{+}
$$

where $\lambda_{m}, \mu_{x}$ and $\varphi_{x}$ are Lagrange multiplier. $\lambda_{m}, \mu_{x}$ and $\varphi_{x}$ are updated

$$
\left\{\begin{array}{c}
\lambda_{x}(t+1)=\left[\lambda_{x}(t)+\xi\left(p_{x}-p_{x}^{\max }\right)\right]^{+} \\
\mu_{m}(t+1)=\left[\mu_{m}(t)+\varsigma\left(p_{x}\left|v_{x m}\right|^{2}-A_{x m}\right)\right]^{+} \\
\varphi_{x}(+1)=\left[\varphi_{x}(t)+\zeta\left(\log \left(1+\gamma_{x}^{\min }\right)-\log \left(1+\gamma_{x}\right)\right]^{+}\right.
\end{array}\right.
$$

where $t$ denotes number of iterations and $[z]^{+}=\max (z, 0), \xi, \zeta$ and $\zeta$ are proper step size.

\subsection{Relay Cooperative Power Allocation Algorithm}

The Lagrangian function of model (13) can described as follows

$$
\begin{aligned}
& L\left\{p_{x}, \beta_{m}, \alpha_{x}, \omega_{x}\right\}=-\sum_{x=1}^{X} \log \left(1+p_{x}\left|h_{x x}\right|^{2} / z_{x}^{2}\right)+\sum_{x} \alpha_{x}\left(p_{x}-p_{x}^{\max }\right) \\
& \quad+\sum_{x} \omega_{x}\left(\log \left(1+\gamma_{x}\right)-\log \left(1+\gamma_{x}^{\min }\right)\right)
\end{aligned}
$$

Let $\partial L / \partial_{x}=0$, the optimization solution can be obtained as follows

$$
p_{x}^{*}=\left(S^{2}\left|h_{x x}\right|^{2} / z_{x}^{2}+\left|g_{x r}\right|^{2} p_{r}^{*}\left|G_{x r}\right|^{2} U\right) / Q W-U
$$

Where

$$
\begin{aligned}
& S=p_{x} z_{x}^{2}\left|g_{x r}\right|^{2}+p_{r}^{*} z_{r}^{2}\left|G_{x r}\right|^{2}+z_{x}^{2} z_{r}^{2} \\
& U=p_{r}^{*} z_{r}^{2}\left|G_{x r}\right|^{2} \quad z_{x}^{2} z_{r}^{2} \\
& W=S^{2}\left(1+p_{x}\left|h_{x x}\right|^{2} / z_{x}^{2}\right)+p_{x}\left|g_{x r}\right|^{2} p_{r}^{*}\left|G_{x r}\right|^{2} \\
& Q=z_{x}^{2}\left|g_{x r}\right| \times 2 \times\left(\alpha+\beta\left|v_{x m}\right|^{2}-\omega\left|h_{x x}\right|^{2} / z_{x}^{2}+\left|h_{x x}\right|^{2} p_{x}\right) .
\end{aligned}
$$

$$
\left\{\begin{array}{c}
(t+1)=\left[\alpha(t)+d t 1 \times\left(p-p_{x}^{\max }\right)\right]^{+} \\
(t+1)=\left[\beta(t)+d t 2 \times\left(p_{x}\left|v_{x m}\right|^{2}-A_{x m}\right)\right]^{+} \\
\omega(t+1)=\left[\omega(t)+d t 3 \times\left(\log \left(1+\gamma^{\min }\right)-\log \left(1+\gamma_{x}\right)\right)\right]^{+}
\end{array}\right.
$$

where tenotes number of iterations. $d t 1$ and $d t 2$ and $d t 3$ represent suitable step size.

The algorithm implements specific steps as follows:

Step 1: Initialization: Set $t=0, \lambda>0, \mu>0, \varphi>0, p_{x}=0$.

Step 2: Calculation: Get the optimal power $p_{x}^{*}$ in non-relay cooperative cognitive system by formula (15).

Step 3: Update: Update the Lagrange multiplier by (16).

Step 4: Return: Go back to step S2 to calculate again until the function converges to a value.

Step 5: Comparison: Calculate the value of inequality (3). If the value of inequality (3) is true, finish the execution of this program. Else, go to next step 6.

Step 6: Re-initialization: set $t=0, \alpha>0, \beta>0, \omega>0, p_{x}>0$.

Step 7: Calculation: Calculate the transmit power $p_{r}^{*}$ of the relay users by formula (12).

Step 8: Update: Update the Lagrange multiplier $\alpha$ and $\beta$ and $\omega$ by formula (23) respectively.

Step 9: Calculation: Get the optimal transmit power $p_{x}^{*}$ by formula (18).

\section{NUMERICAL SIMULATION RESULTS}

The performance of relay collaboration is analyzed by simulation results. Supposed that there are $M=1$ authorized user and $X=5$ cognitive users and the relays $R=5$ are randomly distributed in the network. The channel gain from the transmitter of cognitive user $\mathrm{x}$ to the receiver of cognitive user $\mathrm{x}$ is $h_{x x}=1$. The channel gain $g_{x r}$ of the transmitter of cognitive user $x$ to the relay $r$ is randomly matrix of $5 \times 5$ of a $[1,3]$. The channel gain $G_{r x}$ of the relay $r$ to the receiver of cognitive user x is randomly matrix of $5 \times 5$ of $[0,1]$. The maximum transmit power of cognitive users $\mathrm{x}$ is $p_{x}^{\max }=[1 ; 1 ; 1 ; 1 ; 1]$. The maximum transmit power of relay $\mathrm{r}$ is $p_{r}^{\max }=[2.5 ; 2.5 ; 2.5 ; 2.5 ; 2.5]$. The maximum interference power threshold of cognitive user $\mathrm{x}$ is $A_{x m}=5$. The maximum interference threshold of relay $\mathrm{r}$ is $B_{r m}=1$. The background noise power at cognitive user $\mathrm{x}$ and the relay $r$ is $\sigma_{x}=[0.1955 ; 0.1799 ; 0.1313 ; 0.2011 ; 0.1363]$. The interference $I_{x}$ of the authorized user $\mathrm{m}$ to the cognitive user $\mathrm{x}$ is randomly chosen matrix of $5 \times 1$ from the range of $[0.01,0.02]$. The channel gain between cognitive user $\mathrm{x}$ and authorized user $\mathrm{m}$ is $v_{x m}=0.8$. The channel gain between the relay $\mathrm{r}$ and authorized user $\mathrm{m}$ is $e_{r m}=0.894$. The minimum signal-to-noise ratio is $R=[1 ; 1 ; 1 ; 1 ; 1]$. The average channel gain $d_{x y}$ from the cognitive link $x$ to the cognitive link $y$ is randomly matrix of $5 \times 5$ from the range of $[0.1,0.2]$. 


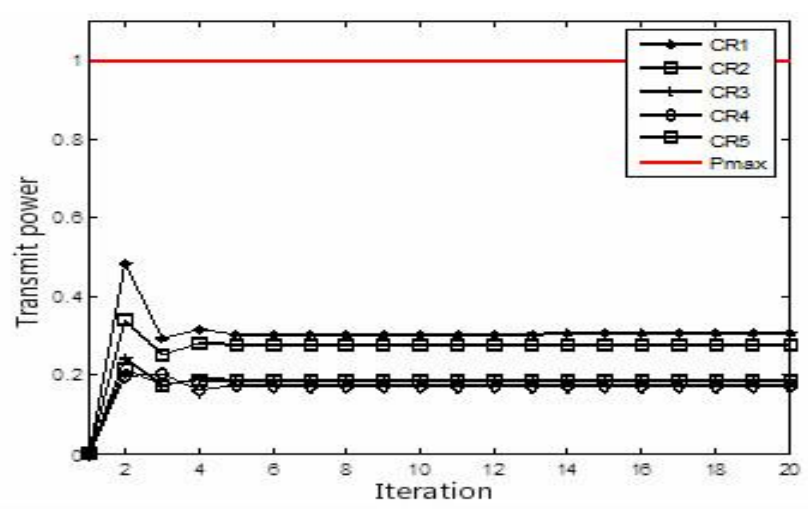

Figure 2: Non-relay cooperative power allocation

Under the non-relay cooperative power allocation, Fig.2 shows that the five cognitive users and cognitive network system reached power allocation and Nash equilibrium respectively. The transmit power of each cognitive user converges and is lower than the maximum transmit power, in which the convergence speed of cognitive user 5 is fastest, the transmit power of the cognitive user 1 is the highest, and the transmit power of the cognitive user 4 is the lowest .

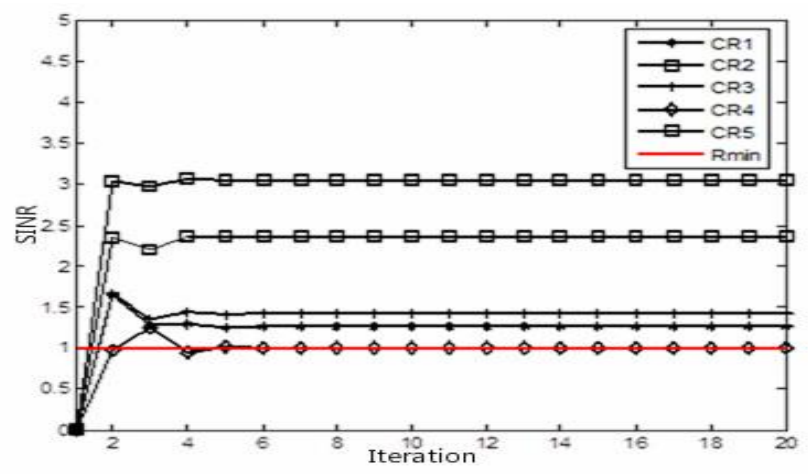

Figure 3: Non-relay signal -to -noise-ratio

Fig.3 presents that each cognitive user obtained SINR. It can be observed that the SINR threshold of the system is 1 . All the five cognitive users obtained SNR greater than the threshold, and all of them can get satisfactory QoS.

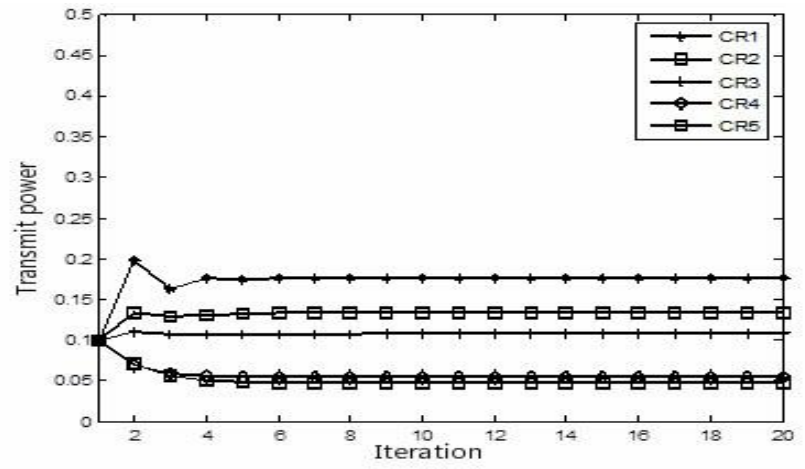

Figure 4: Relay cooperative power allocation

Fig .4 presents the power allocation scheme after the adoption of the relay cooperation, in which the convergence rate and the final transmit power of cognitive user 1 are slower and higher respectively. The convergence rate of the cognitive user 4 is the fastest and the transmit power of the cognitive user 4 is the lowest. The relay cooperative power allocation scheme also achieved the Nash equilibrium.

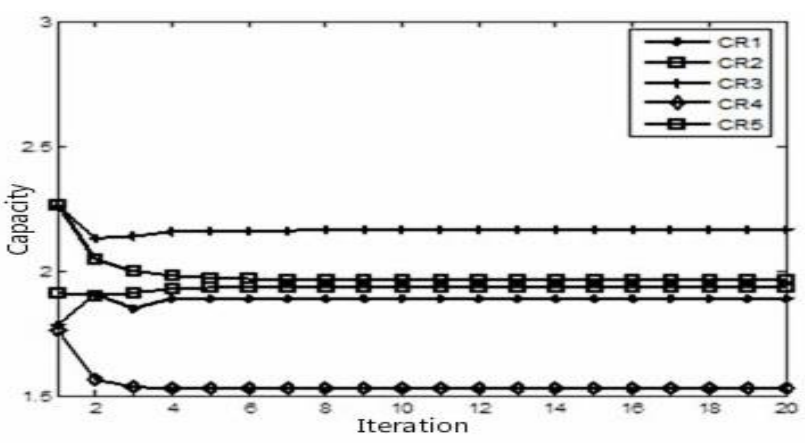

Figure 5: Relay capacity

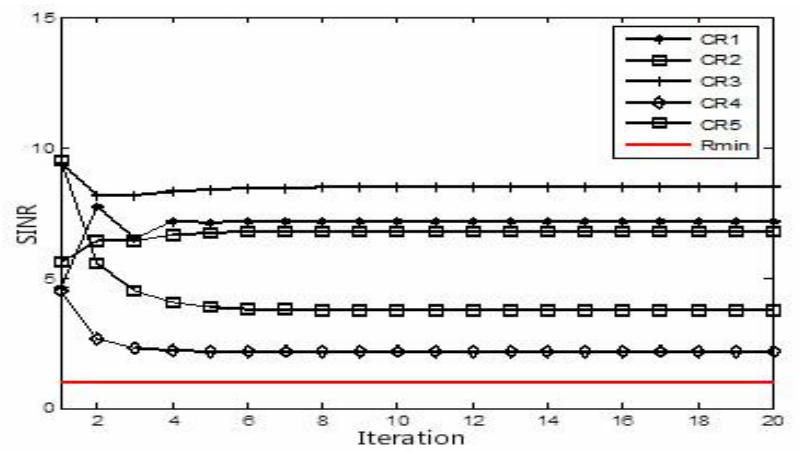

Figure 6: Relay signal -to -noise-ratio

Fig. 5 shows that the cognitive user 3 achieved the maximum capacity, which can better guarantee the communication quality of cognitive user 3 . Fig. 6 shows that the SINR of each cognitive user after the adoption of the relay collaboration, it observed that the SINR of the cognitive user 3 and the cognitive user 4 are lowest and highest respectively. Within the allowable range, the communication quality becomes better as the SINR gets higher, which can better guarantee the cognitive user's QoS.

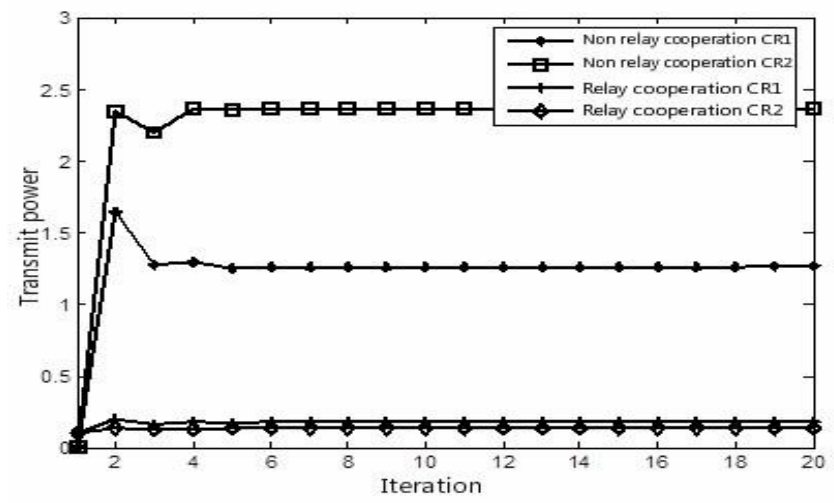

Figure 7: Power allocation contrast

Fig.7 shows that the transmit power of the cognitive user 1 for the nonrelay cooperation is larger than transmit power of the cognitive user 2 for the relay cooperation under the same conditions.

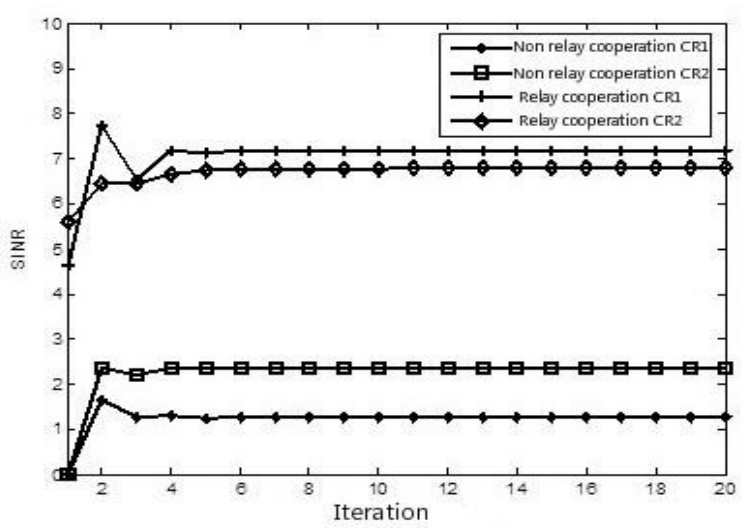

Figure 8: SINR contrast 
Fig.7 and Fig.8 represent the power allocation contrast and SINR contrast of cognitive user 1 and cognitive user 2 respectively. Fig. 8 presents the simulation result of SINR comparison between cognitive user 1 and cognitive user 2 in the two algorithms, which the SINR of the relay collaboration is larger than the SINR of non-relay collaboration, and the SINR of each cognitive user is much than the target SINR.

\section{CONCLUSION}

In cognitive radio, the model is established to maximize the capacity of the system based on shared spectrum and the power allocation algorithm with relay cooperation technology is studied systematically. Simulation results have shown that the SINR of the relay cooperation algorithm is larger than that of the non-relay cooperation algorithm, and the power allocation of the relay cooperation algorithm is superior to that of the non-relay cooperation. It is obvious that the capacity of cognitive network had been increased. It is also shown that the QoS of the authorized users and the cognitive users can be guaranteed respectively.

\section{ACKNOWLEDGMENTS}

The authors would like to thank the Editor and Reviewers for their precious time and efforts in reviewing our paper. This work is supported in part by the National Natural Science Foundation of China under grant no. 61571209 and 61501059, Natural Science Foundation of Jilin (Grant No. 20180101336JC), scientific research fund of Jilin Provincial Education Department (Grant No. 2016134), outstanding youth fund of Jilin City Science and Technology Bureau (Grant No. 20166019), scientific research project of Jilin Institute of Chemical Technology (Grant No. 2015128(D)), and major scientific research project of Jilin Institute of Chemical Technology (Grant No. 2015010).

\section{REFERENCES}

[1] Yuan, F., Guo, Zheng, L. 2012. Resource allocation for relay assisted cognitive radio network with multiple channels [J]. IEEE, 5 (12), 22172221.
[2] Preethams, C. 2015. Power and subchanel allocation for underlay OFDM cognitive networks under interiference temperature [C]. New York: Signale Processing and Comemunication Engineering Systems, 205-209.

[3] Li, G. 2008. Power allocation algorithm for optimal relay cooperative communication system [J]. Electronic journal, 10 (36), 1944-1948.

[4] Xu, Y. 2015. Research on dynamic resource allocation in pad cognitive radio networks [D]. Doctoral disserations. Jilin: Jilin University, 13-15.

[5] Zhu, Y., Chen, J., Tian, Z. 2011. Performance analysis and comparison of multi user collaboration and relay [J]. Communications technology, 01 (44), 75-78.

[6] Alsharoa, A. 2014. Width and power allocation for two-way relaying overlay cognitive radio systems [C]. New York: IEEE Global Communications Conference, 911-913.

[7] Hanan. 2013. Joint power and bandwidths allocation for amplify cooperative communications using stackberg game[J]. IEEE Transaction technology, 4 (62), 1678-1691.

[8] Adian, M.G. 2014. Optimal and sub-optimal resource allocations in multiple input and output orthonal frequency division multiplexing-based multi cooperative cognitive radio networks[J]. IET Communications, 8 (5), 646-650.

[9] Li, M. 2013. Performance analysis and optimization of cooperative spectrum sensing based on relay [J]. Communications technology, 9 (34), 33-40.

[10] Xie, X., Zhang, X., Lei, W. 2011. Research on dynamic selection and handover strategy of cooperative relay in distributed environment [J]. Signal processing, 03 (27), 387-394.

[11] Zhou, M. 2014. Research on resource allocation in cognitive radio system [D]. Doctoral disserations. Jilin: Jilin University, 46-58.

[12] Zhang, X., Zhang, S., Qiu, G. 2012. Fast optimization of detection threshold and relay user configuration [J]. Computer engineering and Application, 48 (24), 117-118. 